\title{
Metabolite Mapping with Extended Brain Coverage Using a Fast Multisection MRSI Pulse Sequence and a Multichannel Coil
}

\author{
Zhengchao Dong, ${ }^{1,2}$ Feng Liu, ${ }^{1}$ Alayar Kangarlu, ${ }^{1}$ and Bradley S. Peterson ${ }^{1}$ \\ ${ }^{1}$ Department of Psychiatry, Columbia University College of Physicians \& Surgeons, New York State Psychiatric Institute, \\ New York, NY 10032, USA \\ ${ }^{2}$ Department of Psychiatry, Columbia University and New York State Psychiatric Institute, 1051 Riverside Drive, No. \#74, New York, \\ NY 10032, USA \\ Correspondence should be addressed to Zhengchao Dong, zhengchaodong@gmail.com
}

Received 28 July 2011; Accepted 16 November 2011

Academic Editor: Eric C. Bourekas

Copyright ( $) 2012$ Zhengchao Dong et al. This is an open access article distributed under the Creative Commons Attribution License, which permits unrestricted use, distribution, and reproduction in any medium, provided the original work is properly cited.

\begin{abstract}
Multisection magnetic resonance spectroscopic imaging is a widely used pulse sequence that has distinct advantages over other spectroscopic imaging sequences, such as dynamic shimming, large region-of-interest coverage within slices, and rapid data acquisition. It has limitations, however, in the number of slices that can be acquired in realistic scan times and information loss from spacing between slices. In this paper, we synergize the multi-section spectroscopic imaging pulse sequence with multichannel coil technology to overcome these limitations. These combined techniques now permit elimination of the gaps between slices and acquisition of a larger number of slices to realize the whole brain metabolite mapping without incurring the penalties of longer repetition times (and therefore longer acquisition times) or lower signal-to-noise ratios.
\end{abstract}

\section{Introduction}

The applications of proton magnetic resonance spectroscopic imaging (1H MRSI) of the brain can benefit from technical developments in pulse sequences and hardware advances to overcome various limitations of MRSI, including low SNR, long acquisition times, and lipid contamination. In pulse sequence development, 2D PRESS-MRSI [1] and 2D STEAM-MRSI [2] have been developed to address several of these limitations and are now widely used. By exciting only a small region of interest within the brain, both sequences permit reduction in the field of view (FOV), which in turn permits a reduction in the number of phase encoding steps and thereby reduces the scan time required to achieve a given spatial resolution [3]. These pulse sequences also reduce contamination of the metabolite signals by lipid signals from the scalp. These and other advantages have motivated the extension of PRESS- and STEAM-MRSI from conventional uses to $3 \mathrm{D}$ or multiple $2 \mathrm{D}$ applications, or to their combination with other techniques, such as echo-planar spectroscopic imaging [4] and spiral MRSI [5]. PRESS and STEAM
MRSI, however, also have several disadvantages, such as relatively small brain coverage and scan times that are still long for human applications, especially when used in 3D or multiple 2D modes [6].

Another development in fast MRSI sequences has been multi-section MRSI [7]. This sequence features two advantages over other PRESS- or STEAM-based fast sequences. (1) Multiple slices are consecutively excited and sampled within one repetition time (TR), whereas lipid signals from subcutaneous fat are suppressed through the application of oblique saturation bands placed by the user on localizer images [3]. (2) Each slice is dynamically shimmed, and therefore better spectral quality can be achieved than with global shimming. In addition, multi-section MRSI also possesses the following features. (1) Full echoes, instead of free induction decays (FIDs), are acquired, so that magnitude spectra can be used without employing phase correction. Acquiring full echoes offers an additional advantage for the reconstruction of MRSI signals sampled using a multichannel receiver RF coil, in that the residual water signals can be used as sensitivity references when combining signals from the various channels 


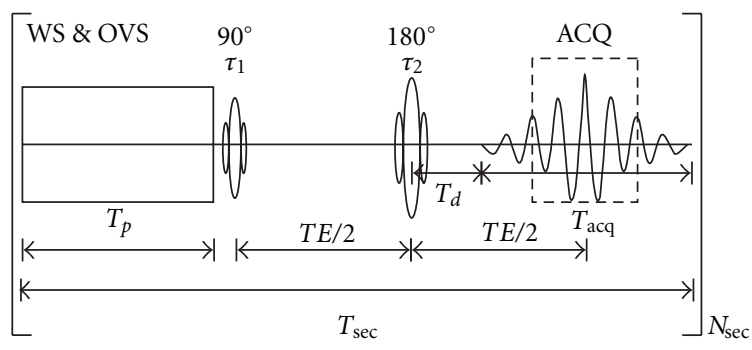

Figure 1: Pulse sequence diagram and timing for multi-section MRSI. The $T_{p}$ is the time for the preparation period, which includes water suppression and outer volume suppression; $\tau_{1}$ and $\tau_{2}$ are the durations of $90^{\circ}$ and $180^{\circ}$ pulses, respectively; TE is the spin echo time; $T_{d}$ is the time delay before data acquisition, which is used for slice selection in the $z$-direction and phase encoding in the $x$ $y$ plane of the slice (not shown); $T_{\mathrm{acq}}$ is the acquisition time; $T_{\mathrm{sec}}$ is the time for one slice, and the total repetition time $T R$ for $N_{\text {sec }}$ slices is $T R=T_{\text {sec }} \times N_{\text {sec }}$. WS: water suppression; OVS: outer volume suppression; ACQ: acquisition.

of the coil [8]. (2) Spacing between slices avoids the signal cancellation caused by "crosstalk" between adjacent slices.

The conventional implementation of multisection MRSI technique [7] also has attendant problems, however. The spacing required between slices fails to acquire information throughout the entire imaging volume, not only losing important information but also creating difficulties for the coregistration of high-resolution MRI images with the MRSI data, which is important for the segmentation of MRSI slices and the analysis of the MRSI data. Although the use of a $288 \mathrm{~ms}$ echo time (TE) is necessary to accommodate the full number of echoes for 512 data points at a sampling rate of $2000 \mathrm{~Hz}$, it also lowers the SNR and requires a long TR of 2.3 seconds to sample phase-encoded signals from 4 slices. Because each TR is "full" (Figure 1), any further increase in the number of slices is possible only by linearly increasing the $T R$, which undermines the "fast" feature of the pulse sequence. The limited number of slices also limits the volume coverage, requiring an increase in slice thickness and a tradeoff with compromising spatial resolution to cover a larger volume with the same 4 slices.

Hardware development, and the use of multichannel coils in particular, has benefitted the applications of $1 \mathrm{H}$ MRSI. The initial and conventional application of multichannel coils in MRS was to improve SNR [9-12], as the array of surface coils provides superior sensitivity compared with more conventional quadrature volume coils [10]. In recent years, parallel MRSI has employed multichannel coils to accelerate data acquisition [13]. The gains in SNR or the reduction in scan time, though valuable in their own right, can be further traded for other potential advantages, such as spatial resolution of MRSI [14].

We note that the limitations of multi-section MRSI are not caused by intrinsic disadvantages of the sequence, but rather are the consequence of technical limitations and compromises that can be addressed powerfully using multichannel coil technology. We hypothesize that the use of multichannel coil technology for multi-section spectroscopic im- aging can eliminate the need for gaps between slices and permit an increase in the number of slices without resorting to longer acquisition times or lower spatial resolution for improved brain coverage. Therefore, our aim in this report is to synergize the multi-section spectroscopic imaging (SI) pulse sequence [7] with multichannel coil technology to realize whole brain metabolite mapping by removing the gaps between slices and increasing the number of slices without resorting to trading off longer TRs and therefore longer acquisition times or poorer spatial resolution for more brain coverage.

\section{Methods}

2.1. MRSI Sequence. The timing of the simplified RF pulse sequence for multi-section MRSI is schematically shown in Figure 1. $T_{p}$ is the time for the preparation period, which includes water suppression and outer volume suppression; $\tau_{1}$ and $\tau_{2}$, are the durations of $90^{\circ}$ and $180^{\circ}$ pulses, respectively; $T E$ is the spin echo time; $T_{d}$ is the time delay before data acquisition, which is used for slice selection in the $z$-direction and phase encoding in the $x-y$ plane of the slice (not shown); $T_{\text {acq }}$ is the acquisition time; $T_{\text {sec }}$ is the time for one slice; the total repetition time $T R$ for $N$ slices is $T R=T_{\text {sec }} \times N_{\text {sec }}$. The timing of the sequence is determined by hardware capacities and practical considerations. The times of $T_{p}$ and $T_{d}$, as well as $\tau_{1}$ and $\tau_{2}$ for example, relate to the hardware and are optimized to the shortest possible times during the design of the pulse sequence. They are treated as constants in this application. $T_{\text {acq }}$ and $N_{\text {sec }}$ are variables selected by the users. $T_{\text {acq }}$ equals N/SW, where $N$ is the number of data points in the echo and SW is the spectral width, both of which are determined by practical considerations and compromises. For example, one way to reduce scan time, $T_{\mathrm{sec}}$, is to reduce the $T E$, which is possible by reducing $T_{\text {acq. Increasing spectral }}$ width can reduce $T_{\text {acq }}$,but does so at the expense of increasing noise. Reducing $N$ reduces $T_{\text {acq }}$, but it will reduce the spectral resolution and produce truncation effects, as the acquisition may begin after the echo signal is fully built up and end before the echo signal is fully decayed, as shown in the dashed box in Figure 1. Although zero-padding the truncated echo prior to Fourier transformation may improve the spectral resolution, it will also produce wiggling in the spectrum. Both low digital resolution and wiggling will hinder spectral fitting in the frequency domain [15]. All these factors considered, SW and $N$ were conventionally designated to be $1000 \mathrm{~Hz}$ and 256, respectively, for a $1.5 \mathrm{~T}$ scanner [7] and $2000 \mathrm{~Hz}$ and 512 for 3T scanners. With these parameters, and for $P E=32 \times 32$ and $N_{\mathrm{sec}}=4$, the $T E$ is $280 \mathrm{~ms}$, yielding a $T_{\mathrm{sec}}$ of $575 \mathrm{~ms}, T R=2300 \mathrm{~ms}$, and a scan time of 30 minutes. Together with preparation time, including slice and outer volume suppression (OVS) band prescription, shimming, and prescanning, the scan time totals $50 \mathrm{~min}$. Further increasing the number of slices will proportionally increase the scan time.

We reduced the number of data points in the echoes acquired on a $3 \mathrm{~T}$ scanner from 512 to 256 , so that 7 slices will be covered in a TR of $2300 \mathrm{~ms}$. To avoid the disadvantage of 
low spectral resolution and the truncation effects on spectral fitting in the frequency domain associated with acquisition of a limited number of data points, we adopted an algorithm of spectral fitting in the time domain. Those details will be described below under Data Processing section.

2.2. Computer Simulation. We performed computer simulations to compare the effects of spectral fitting algorithms on signal truncation in the time domain and spectral resolution or sinc wiggles in the frequency domain. The signal simulated the 3 singlets of $\mathrm{Ch}, \mathrm{Cr}$, and NAA with amplitudes of 24, 36, and 48 (a.u.), respectively. The linewidth was $10 \mathrm{~Hz}$ for either a Lorentzian or Gaussian lineshape. For a Voigtian lineshape, the Lorentzian decay was $2.5 \mathrm{~Hz}$, and the Gaussian decay was $7.5 \mathrm{~Hz}$ for all three lines. Spectral width was $2000 \mathrm{~Hz}$, the number of data points in the echo was 256, and it was zero-filled to 512 or 1024 . We also conducted a Monte Carlo simulation for signal fitting in the time domain and compared the standard deviations of the estimated amplitudes with their Cramer Rao Lower Bounds (CRLBs), a benchmark for assessing the accuracy of spectral fitting algorithms.

2.3. MR Data Acquisition. We carried out all MR measurements on a spectroscopic phantom and on 3 human volunzteers, respectively, using a whole body 3T scanner (Signa HDx 3.0T, GE Healthcare, Waukesha, WI), equipped with a quadrature transmit/receive head coil, and an 8-channel receive-only head coil. First, we acquired scout images using a commercial gradient recalled echo-based 3-planar MRI sequence and then prescribed the localizer images of the MRSI slices, which were in axial plane for phantom scans and in an oblique axial plane parallel to the anterior commissure-posterior commissure line in human subjects. Then, we localized the MRSI slices by copying the location of the localizer images and acquired MRSI data using the multiplanar MRSI pulse sequence [7]. The number of slices, the slice thickness, and spacing varied in accord with those of different MRSI sessions (vide infra). However, typical parameters of the MRSI pulse sequence were as follows for both the phantom and human subjects: number of slices $=7$; slice thickness $=10 \mathrm{~mm}$; spacing between slices $=4$ or $0 \mathrm{~mm}$; nominal number of phase encodings $(\mathrm{PEs})=16 \times 16$ or $32 \times 32 ; T R / T E=2300 / 144 \mathrm{~ms} ;$ spectral width $=2000 \mathrm{~Hz}$; number of data points in the echo $=256$. The duration of an MRSI scan was 8 or 30 minutes, depending on the number of PEs. Total scan time including MRI localizer, MRSI slice prescription, OVS band placement, and field shimming was about 26 or 50 minutes. When repeating the MRSI scans with different slice spacings, we changed only the spacing but not the position of the central (4th) slice. Therefore, we used signals from this slice to assess the effects of differing slice spacings on the "crosstalk" between slices. For each MRSI scan with a differing spacing between slices, we performed an autoprescan for field shimming and transmitter gain optimization. Then receiver gains (RGs) were manually adjusted if needed to retain the same RG values for all MRSI scans. The protocol was approved by the
Institutional Review Board of the New York State Psychiatric Institute. Written informed consent was obtained from each human participant.

\subsection{Data Processing}

2.4.1. Combination of Multichannel Data. The $k$-space MRSI data from individual coil elements were transformed to the image domain using a 2D spatial Fourier transform after spatial filtering that used a Hamming window function. The data from the multiple coil elements were combined using the following procedures.

(1) Water Signal Removal. We used a matrix-pencil-methodbased procedure [16] to decompose the signal, identify water components by their frequencies, and remove them from the signal [16]. This method was able to remove water signal almost completely (>98\%) without interfering with the metabolite signals of interest.

(2) Removal of Corrupted Points. We replaced the first 6 echo data points [17], which were corrupted by the activation of the analogue-to-digit converter, with 6 extrapolated points derived from signal parameters that were estimated from the uncorrupted data points using the matrix pencil method.

(3) Data Apodization. We next multiplied the cleaned echo data by a Gaussian function, $G(t)=e^{-\beta t^{2}}$, to suppress noise and reduce baseline distortion, albeit at the expense of line broadening. The line broadening was $10 \mathrm{~Hz}$ for phantom data and $4 \mathrm{~Hz}$ for in vivo data.

(4) Phase Alignment. We eliminated voxelwise phase differences in echoes from individual coil elements by subtracting the phases at the top of their echoes.

(5) Weighted Summation. We summed the phase-aligned echoes using weighting factors that were proportional to the echo amplitudes and inversely proportional to the noise levels of the coil elements. Noise levels were determined by measuring the standard deviations of the data points in the signal-free regions of the frequency domain spectra of a phantom.

2.4.2. Spectral Fitting. We quantified the spectral components using the following general model function to fit the echoes:

$$
S(t)=\sum_{m=1}^{M} A_{m} e^{i\left(2 \pi f_{m} t+\varphi_{m}\right)} e^{-\alpha_{m}|t|-\beta_{m} t^{2}}
$$

where $A_{m}, f_{m}, \varphi_{m}$, and $\alpha_{m}$ represent the amplitude, frequency, phase, and Lorentzian decay of peak $m$, respectively; $\beta_{m}$ is the Gaussian decay. Note that the $t$ runs from $-\mathrm{dt} \cdot N / 2$ to $\mathrm{dt}$. $(N / 2-1)$, where dt is dwell time and $N$ is the number of data points in the echo. Note also that when fitting the spectrum with a pure Lorentzian model, we set $\beta_{m}$ to be zeros; when 
TABLE 1: Comparisons of signal amplitudes acquired on a phantom with or without spacing between slices. Means and SD were calculated using (2). $n$ is the number of selected voxels in the 4 th slice. The $T R$ was $2.3 \mathrm{~s}$ for tests 1 and 2 , and $3.0 \mathrm{~s}$ for test 3 . The labeled concentrations for NAA, Cr, and Cho were 12.5, 10.0, and $3.0 \mathrm{mM}$, respectively.

\begin{tabular}{lccc}
\hline Test & $\begin{array}{c}\text { NAA } \\
(\text { mean } \pm \text { SD })\end{array}$ & $\begin{array}{c}\text { Cr } \\
(\text { mean } \pm \mathrm{SD})\end{array}$ & $\begin{array}{c}\text { Cho } \\
(\text { mean } \pm \text { SD })\end{array}$ \\
\hline $1(n=47)$ & $1.26 \% \pm 4.16 \%$ & $1.71 \% \pm 3.23 \%$ & $1.05 \% \pm 5.82 \%$ \\
$2(n=39)$ & $0.51 \% \pm 4.22 \%$ & $0.07 \% \pm 6.15 \%$ & $-0.47 \% \pm 4.17 \%$ \\
$3(n=49)$ & $0.47 \% \pm 3.29 \%$ & $-1.73 \% \pm 4.43 \%$ & $-0.84 \% \pm 4.82 \%$ \\
\hline
\end{tabular}

fitting the spectrum with pure Gaussian model, we set the $\alpha_{m}$ to be zeros; when fitting the spectrum with a Voigtian model, we set the $\beta_{m}$ to be the same for all $M$ peaks.

Further notes are warranted for the process of spectral fitting. The signal parameters in (1) were determined using a nonlinear least squares fitting routine in Matlab (2007a, The MathWorks, Natick, Massachusetts). To ensure that these parameters were real numbers, the real parts and imaginary parts of both the model function in (1) and the measured echo signal were concatenated, respectively, to form realnumber series. It is important to determine accurately the initial values for $A_{m}, f_{m}$, and $\alpha_{m}$, as well as the number of peaks, to ensure the robustness of the fitting. They were estimated from the magnitude spectrum obtained by zeropadding the echo to 4096 points and then performing FFT, whereas the global initial phase was obtained by the phase of the top point of the echo.

2.4.3. Comparing Data Acquired with or without Slice Spacing. The influence of acquiring contiguous slices on signal intensity was determined by comparing signal amplitudes from the two scans with or without spacing between slices. We selected voxels from a region within the brain in the 4th slice whose location was the same for the scans with or without spacing. The amplitudes of the $m$-th signal components (peak areas in the frequency domain), $A_{m}$, were used to calculate the relative differences of the signals obtained with or without spacing

$$
d_{m}=\frac{\left(A_{m, w \cdot s p}-A_{m, \mathrm{wo} \cdot \mathrm{sp}}\right)}{A_{m, w \cdot \mathrm{sp}}} .
$$

We used the means and standard deviations (S.D.) of the $d$ 's to evaluate the reductions in signal caused by the "crosstalk" introduced between slices when spacing was removed.

\section{Results}

3.1. Comparing Signals with and without Spacing between Slices. Tables 1 and 2 show the relative differences of the amplitudes, calculated using (2), of the phantom and in vivo MRS signals acquired with and without spacing between slices. The phantom data did not show significant differences in these values (Table 1). The mean values of the differences in amplitudes (or the signal reduction) caused by the
TABLE 2: Comparisons of signal amplitudes acquired on a human subject with or without spacing between slices. Means and SD were calculated using (2). $n$ is the number of selected voxels in the 4 th slice. The TR was $2.3 \mathrm{~s}$ for tests 1 and 2 , and $3.0 \mathrm{~s}$ for test 3 .

\begin{tabular}{lccc}
\hline Test & $\begin{array}{c}\text { NAA } \\
(\text { mean } \pm \text { SD })\end{array}$ & $\begin{array}{c}\text { Cr } \\
(\text { mean } \pm \text { SD })\end{array}$ & $\begin{array}{c}\text { Cho } \\
(\text { mean } \pm \text { SD })\end{array}$ \\
\hline $1(n=34)$ & $2.50 \% \pm 4.47 \%$ & $7.30 \% \pm 4.09 \%$ & $2.94 \% \pm 9.71 \%$ \\
$2(n=35)$ & $7.33 \% \pm 4.24 \%$ & $1.56 \% \pm 6.23 \%$ & $5.63 \% \pm 6.64 \%$ \\
$3(n=38)$ & $2.80 \% \pm 5.95 \%$ & $3.04 \% \pm 8.07 \%$ & $2.52 \% \pm 10.0 \%$ \\
\hline
\end{tabular}

TABLE 3: Relative errors (\%) of spectral fitting of the simulated signal in the frequency domain, caused by truncation effects and by wiggles, respectively.

\begin{tabular}{lcccccc}
\hline \multirow{2}{*}{ Lineshape } & \multicolumn{3}{c}{ Original 256 points } & \multicolumn{4}{c}{ Zero-padding to 1024 points } \\
& NAA & Cr & Ch & NAA & Cr & Ch \\
\hline Lorentzian & 4.72 & 2.04 & 4.34 & 4.29 & 3.23 & 0.82 \\
Gaussian & 4.06 & 0.19 & 1.72 & 4.38 & 2.10 & 0.44 \\
Voigtian & 8.45 & 15.31 & 6.07 & 7.00 & 9.33 & 0.59 \\
\hline
\end{tabular}

"crosstalk" in the in vivo data was $<7.5 \%$ for the first two tests, when $T R=2.3 \mathrm{~s}$. On the average, the signal reduction was smaller for the third test when $T R=3.0 \mathrm{~s}$ (Table 2).

\subsection{Comparison of Spectral Fitting in the Time and Frequency} Domains. Errors can be as large as $4 \%$ when fitting the noise-free original data in the frequency domain with a Lorentzian or Gaussian lineshape, or up to $15 \%$ for fitting data with a Voigtian lineshape (Table 3). These errors are the consequence of low spectral resolution and a (Voigtian) model mismatch in the frequency domain. When fitting the zero-padded signals (from the original 256 to 1024 points) in the frequency domain, errors were of the same order, whereas errors were reduced to zero when fitting the spectrum with the original 1024 points. This finding indicates that errors using padded signals are caused by wiggles (Figure 2). In contrast, when fitting the original 256 points of noise-free signals in the time domain, the signals can be perfectly recovered, regardless of whether the line shapes are Lorentzian, Gaussian, or Voigtian. Monte Carlo simulations with 400 noise realizations added to the 256 points time domain signals revealed that the estimated amplitudes of NAA, $\mathrm{Cr}$, and $\mathrm{Ch}$ approximated the true values for all three lineshapes. The SDs of the Lorentzian and Voigtian lineshapes were less than 2 times the Cramér-Rao Lower Bounds (CRLBs), whereas the SDs of the Gaussian line shapes were approximately 1.5 times the CRLB (Table 4).

3.3. Combination of Multichannel Signals. The phase differences caused by the differing positions of coil elements were eliminated, yielding perfect alignment of the array signals in the frequency domain and thereby enhancing SNR (Figure 3).

3.4. Spectral Fitting and Metabolite Maps. The performance of spectral fitting of the data of 256 points in the time domain 

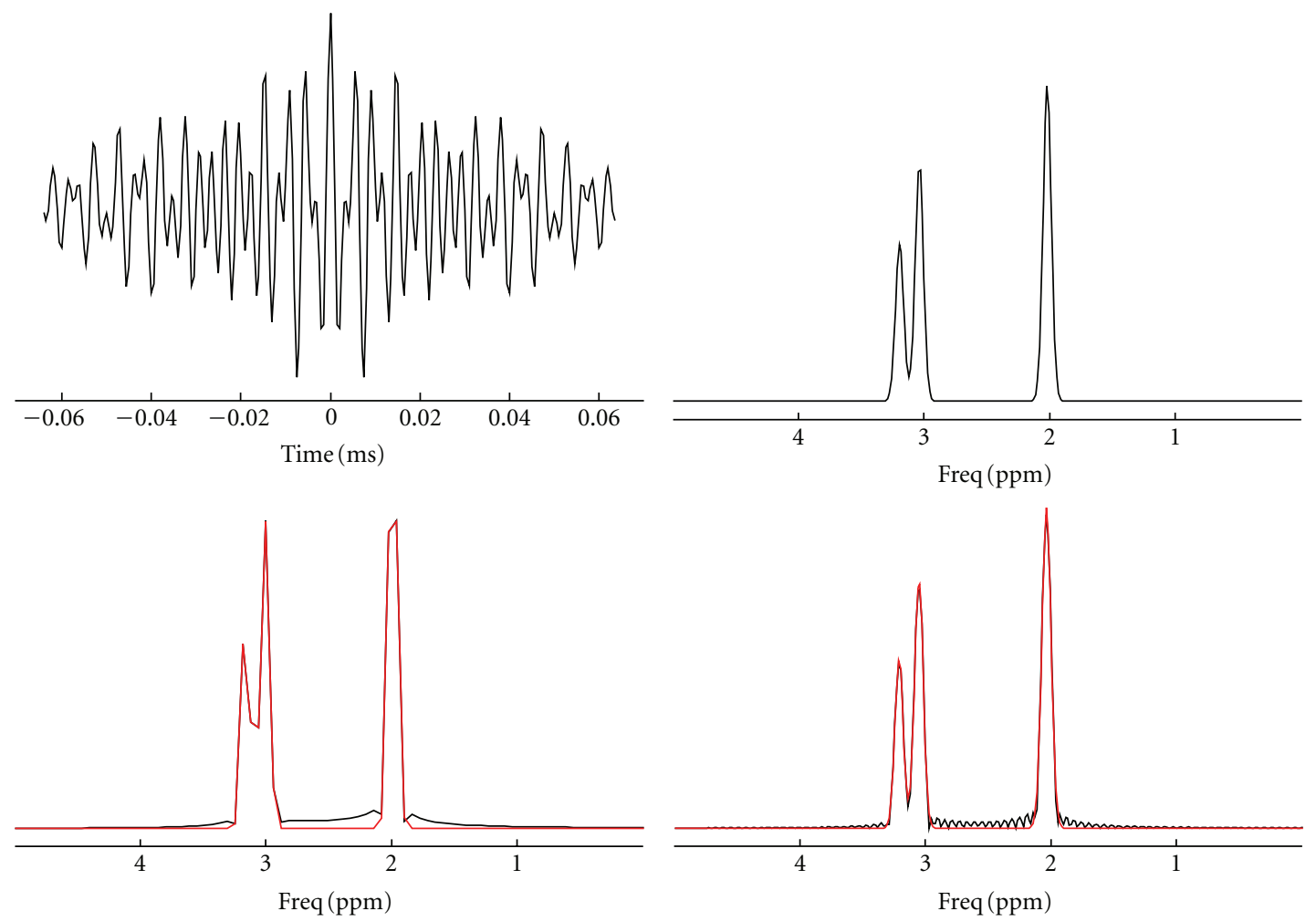

FIGURE 2: Simulated echo with 256 data points and its spectrum obtained with 1024 data points (upper). The time and frequency domain fitting algorithms can fully recover each of them. However, when fitting the spectrum with 256 data points, or when the spectrum was zerofilled from 256 to 1024 data points, sizeable errors are evident as a consequence of either low spectral resolution or truncation effects, which manifest as wiggles in the spectrum after zero-filling (lower).

Table 4: Results of Monte Carlo study of the estimated amplitudes (mean $\pm \mathrm{SD}$ ). The true values of NAA, Cr, and $\mathrm{Ch}$ are 48, 36, and 24, respectively; the CRLBs are $0.1574,0.1288$, and 0.1858 for Lorentzian, Gaussian, and Voigtian, respectively.

\begin{tabular}{lccc}
\hline Lineshape & NAA & Cr & Ch \\
\hline Lorentzian & $47.99 \pm 0.27$ & $35.99 \pm 0.31$ & $24.02 \pm 0.30$ \\
Gaussian & $48.00 \pm 0.18$ & $36.00 \pm 0.19$ & $24.00 \pm 0.19$ \\
Voigtian & $48.00 \pm 0.37$ & $36.00 \pm 0.40$ & $24.00 \pm 0.38$ \\
\hline
\end{tabular}

is further demonstrated using in vivo data. An example of the spectral fitting is shown in Figure 4, in the form of an absolute spectrum. The whole brain MRSI of NAA is shown in Figure 5, overlaid on their localizer images. Similar results were obtained for the other two subjects.

\section{Discussion}

We reported herein improvements of the well-known and widely used multi-section MRSI technique. The modified pulse sequence has several important advantages over its standard implementation: (1) it eliminates spacing between slices; (2) it allows increase in the number of slices (in our study from 4 to 7 ) without the expense of increasing scan time; (3) it permits a reduction in slice thickness and therefore improves spatial resolution, which can be achieved without the expense of reducing overall volume coverage because of the increased number of slices that are acquired; (4) it employs a multichannel coil for data acquisition to improve SNR. It also employs fitting of the severely truncated full echo in the time domain. These improvements make the multi-section MRSI technique more diagnostically valuable.

Compared with the performance of the original MRSI pulse sequence, each modification individually can have its own unique advantages, limitations, and challenges. Spacing between slices, for example, was introduced in the original implementation of the sequence to avoid "crosstalk" between adjacent slices, a phenomenon of signal interference caused by the imperfect slice profiles in which edges are not clearcut but instead interlace with one another. "Crosstalk" can cause signal loss and thus reduce SNR. Slice spacing, however, comes at the expense of information loss from the volumes between slices that are not imaged, which can be as large as $30 \%$ of the total MRS imaging volume. This reduced volume of coverage also reduces overall spatial resolution. The reduction in signal caused by eliminating spacing between slices was less than $10 \%$ in vivo using our modified MRSI sequence (Table 2). This loss of signal would be regarded as substantial if the SNR of the original signal was low, but it is in fact an inconsequential loss when using an 8-channel multichannel coil array, which typically doubles SNR compared with use of a standard quadrature coil $[8,11,12]$. Therefore, even with a $10 \%$ signal reduction 

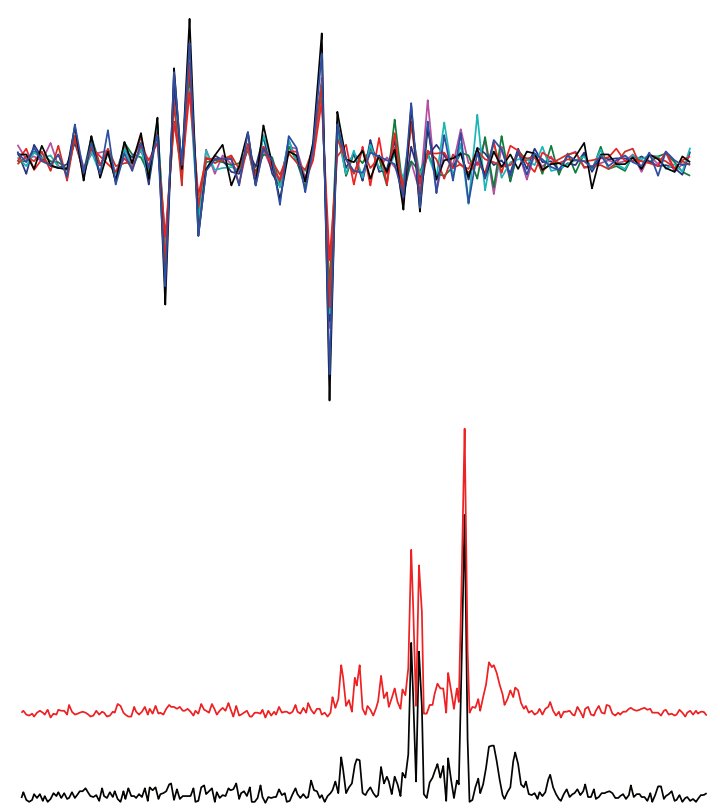

Figure 3: Examples of the combination of in vivo signals from a multichannel coil array. Upper: phase alignment displays the real parts of the spectra without phase correction. Lower: a combined spectrum (red) and a channel spectrum with the highest SNR, shown in absolute mode. The SNRs of the combined spectrum and the channel spectrum with the highest SNR were 77.8 and 46.1, respectively.

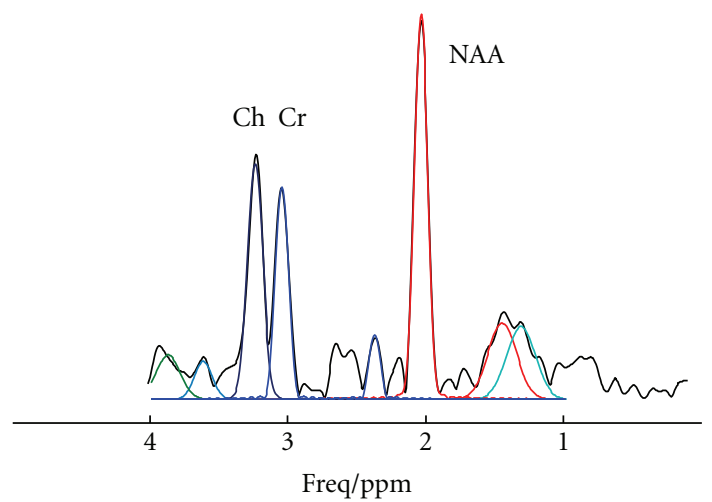

Figure 4: An example of spectral fitting of the in vivo data in the time domain for 256 data points, displayed in the frequency domain and absolute mode. Shown in colors are the individual fitted spectral lines overlaid on the measured spectrum in black. The excellent fitting supports the merit of spectral fitting in the time domain.

caused by "crosstalk" from contiguous slices, the SNR using a multichannel coil in conjunction with our modified sequence is still much higher than the SNR when using a standard quadrature head coil without "crosstalk" (Figure 4). We note, in fact, that increasing the number of slices from 4 to 7 can actually help to reduce the "crosstalk" across slices, because interleaved slice excitations are separated temporally by at most one interval if the number of slices is 4 (i.e., 1-32-4), whereas with 7 slices, the temporal interleave can be 2 or 3 intervals for adjacent slices (e.g., 1-3-5-7-2-4-6, where slices 1 and 2 are temporally separated by 3 intervals, slices 4 and 5 are separated by 2 intervals, and so on). In addition, the reduction in slice thickness theoretically would lead directly to a proportional reduction in SNR when shimming is perfect. In reality, however, and especially in regions near air-tissue interfaces, the effect of reduced volumes in each slice on SNR is not linear because smaller volumes come with narrower line widths. Consequently, we did not observe significant signal drop-out in regions of air-tissue interface within the lower frontal lobe (Figure 5). Thus, the advantages that our modified multi-section MRSI sequence provides are the whole brain coverage with contiguous slices, potentially improved spatial resolution, and improved spectral line width.

The reduction in number of data points in the echo, which is the core of the current modification, poses severe challenges for spectral fitting and spectral quantification. Computer simulation (Table 3 ) showed that fitting a spectrum with low spectral resolution in the frequency domain can produce sizeable errors. Conversely, increasing the spectral resolution can improve the accuracy of spectral fitting in the frequency domain. When the number of data points in the original echo was 1024, the frequency domain fitting algorithm perfectly recovered the spectrum. However, significant errors remained if the spectrum was obtained by zero-filling the 256 data points to 1024 (Table 3), reflecting the detrimental effects of truncation or wiggle artifacts on spectral fitting. Spectral fitting in the time domain, in contrast, accurately fits the echo using 256 data points, suggesting that time domain spectral fitting is preferable for signals with fewer data points, a possibility that Monte Carlo simulation of the signal (Table 4) and spectral fitting of the in vivo data (Figures 4 and 5) are verified.

Our implementation of whole brain metabolite mapping using a multiple 2D MRSI sequence also affords distinct advantages over 3D MRSI, in which the number of phase encoding (PE) steps in the 3rd dimension is typically 8 [18, 19]. This smallv number of data points entails pronounced, long-range signal contamination across slices because of the effects of the point spread function when reconstructing the slice data directly using FFT. Spatial filtering with Hamming, Hanning, or Kaiser window functions must be applied prior to FFT to suppress this signal contamination, but at the expense of increasing the amount of signal bleeding between adjacent slices. Therefore, the effective slice thickness of $3 \mathrm{D}$ MRSI is approximately 1.4 times that of the nominal slice thickness, which significantly degrades spatial resolution. The effective thickness of slices using multiple 2D MRSI, on the other hand, is close to its nominal value, despite the fact that the slice profile is not ideal and slight "crosstalk" is present between slices. Another limitation of 3D MRSI using PRESS localization is that the first and last slices cannot be used, reducing the effective number of slices by $2[18,19]$. Our pulse sequence, in contrast, provides high-quality MRSI images in all 7 slices (Figure 5). 3D MRSI has a distinct advantage over multi-section MRSI, however, in that the location of slices in 3D MRSI can be shifted to specific 


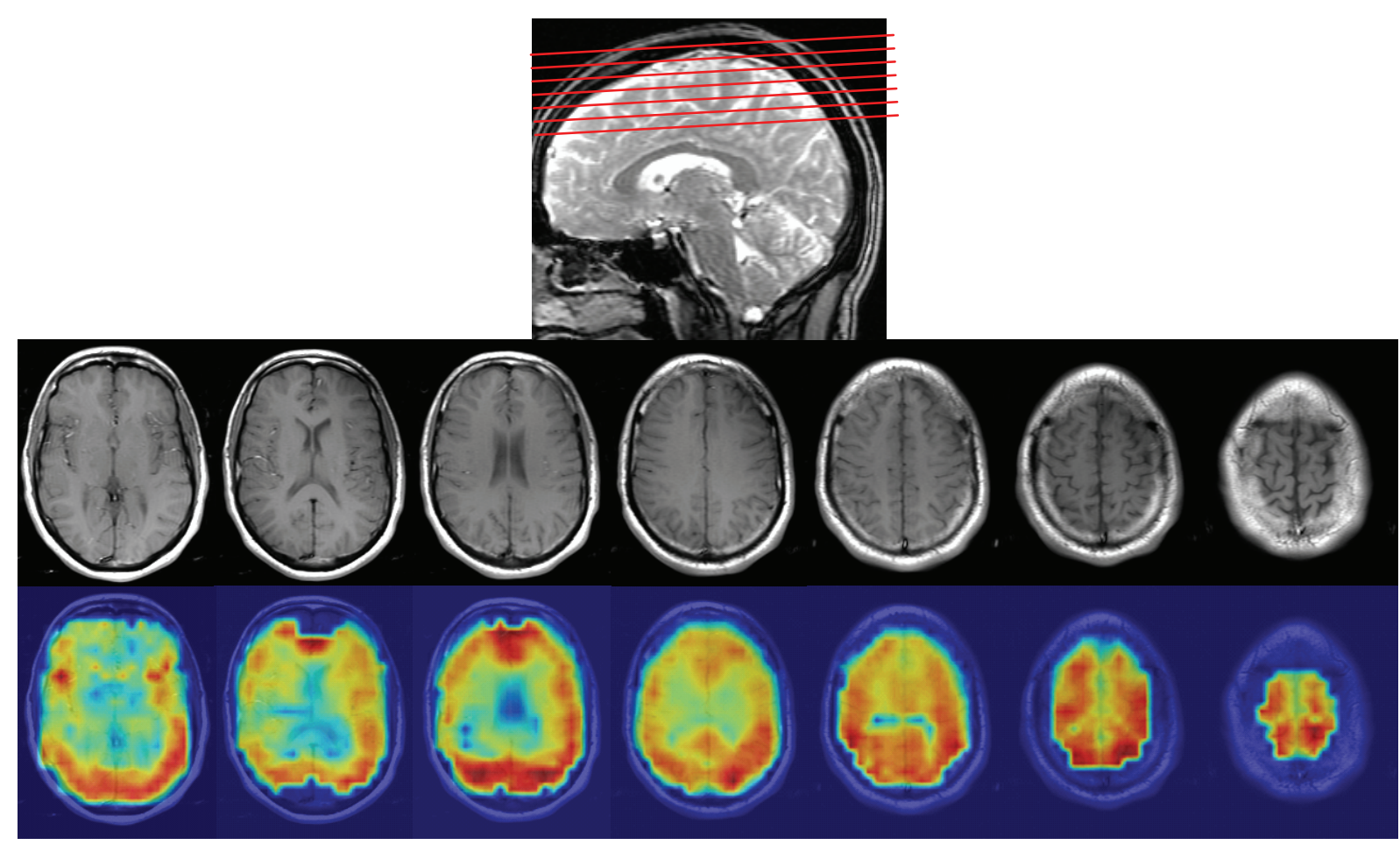

FIGURE 5: Slice prescription (top), localizer images (middle), and NAA images overlaid on localizers (bottom). The slices are contiguous and cover a large volume of the brain.

anatomical regions of interest by employing a phase shift of the Fourier transform.

Comparing signal losses in phantom and in vivo MRSI data in the absence of spacing between slices showed that signal loss caused by "crosstalk" is related to the slice profile, the spatial and temporal separations between successive excitations, and the longitudinal relaxation times of the subjects. Our experimental procedures ensured that the spatial and temporal separations of excitations were identical for the phantom and in vivo scans. The longitudinal relaxation times of molecules in the phantom were significantly longer than those in the human brain, and therefore comparatively larger signal loss could be expected if all other conditions remained the same. The fact that signal loss was negligible in the phantom (Table 1) but was approximately $10 \%$ in the human brain (Table 2) when spacing was eliminated as compared with $4 \mathrm{~mm}$ spacing can be attributed to a better slice profile in the phantom. Comparing spectral fitting in the time domain with fitting in the frequency domain has been of long-standing interest in MRS [2025]. As the time domain and frequency domain signals are related by the Fourier transform, they theoretically have the same information content. However, fitting in the time and frequency domains may have their unique advantages and disadvantages depending on the properties of the signal, such as noise level and phase or baseline distortions [20-25]. Time domain methods are preferable in the presence of distortions in the measured signals, including truncation [20,23]. Our computer simulation provided a numerical example for the detrimental effects of truncation in the frequency domain and showed that fitting in the time domain is immune to those effects.

In conclusion, we have presented a realization of extended brain metabolite mapping using a multiple 2D MRSI pulse sequence in conjunction with use of a multichannel RF coil and spectral fitting in the time domain. These combined techniques have permitted an increase in the number of slices from 4 to 7 , without sacrificing scan time or SNR. The extended brain coverage, reduced slice thickness, and increased SNR can potentially make the sequence more clinically valuable.

\section{Grant Support}

This study was funded in part by NIMH grants K02 74677, MH089582, MH068318, 1P50MH090966, NIEHS grant ES015579, and NIDA grants DA027100, and DA017820.

\section{Acknowledgments}

The authors would like to thank Drs. Jan Willem van der Veen and Dikoma Shungu for their help in updating the multi-section MRSI sequence to allow the acquisition of more than 4 slices. They would like to thank the anonymous reviewers for their insightful comments and suggestions that help improve the paper. 


\section{References}

[1] P. Bottomley, Selective Volume Method for Performing Localized NMR Spectroscopy, 1984.

[2] J. Frahm, H. Bruhn, M. L. Gyngell, K. D. Merboldt, W. Hanicke, and R. Sauter, "Localized high-resolution proton NMR spectroscopy using stimulated echoes: initial applications to human brain in vivo," Magnetic Resonance in Medicine, vol. 9, no. 1, pp. 79-93, 1989.

[3] P. B. Barker and D. D. M. Lin, "In vivo proton MR spectroscopy of the human brain," Progress in Nuclear Magnetic Resonance Spectroscopy, vol. 49, no. 2, pp. 99-128, 2006.

[4] S. Posse, C. DeCarli, and D. Le Bihan, "Three-dimensional echo-planar MR spectroscopic imaging at short echo times in the human brain," Radiology, vol. 192, no. 3, pp. 733-738, 1994.

[5] E. Adalsteinsson, P. Irarrazabal, S. Topp, C. Meyer, A. Macovski, and D. M. Spielman, "Volumetric spectroscopic imaging with spiral-based k-space trajectories," Magnetic Resonance in Medicine, vol. 39, no. 6, pp. 889-898, 1998.

[6] M. L. Zierhut, E. Ozturk-Isik, A. P. Chen, I. Park, D. B. Vigneron, and S. J. Nelson, "1H spectroscopic imaging of human brain at 3 Tesla: comparison of fast three-dimensional magnetic resonance spectroscopic imaging techniques," Journal of Magnetic Resonance Imaging, vol. 30, no. 3, pp. 473-480, 2009.

[7] J. H. Duyn, J. Gillen, G. Sobering, P. C. M. Van Zijl, and C. T. W. Moonen, "Multisection proton MR spectroscopic imaging of the brain," Radiology, vol. 188, no. 1, pp. 277-282, 1993.

[8] Z. Dong and B. Peterson, "The rapid and automatic combination of proton MRSI data using multi-channel coils without water suppression," Magnetic Resonance Imaging, vol. 25, no. 8, pp. 1148-1154, 2007.

[9] T. Schäffter, P. Börnert, C. Leussler, I. C. Carlsen, and D. Leibfritz, "Fast $1 \mathrm{H}$ spectroscopic imaging using a multi-element head-coil array," Magnetic Resonance in Medicine, vol. 40, no. 2, pp. 185-193, 1998.

[10] S. M. Wright and L. L. Wald, "Theory and application of array coils in MR spectroscopy," NMR in Biomedicine, vol. 10, no. 8, pp. 394-410, 1997.

[11] M. A. Brown, "Time-domain combination of MR spectroscopy data acquired using phased-array coils," Magnetic Resonance in Medicine, vol. 52, no. 5, pp. 1207-1213, 2004.

[12] T. Prock, D. J. Collins, A. S. K. Dzik-Jurasz, and M. O. Leach, "An algorithm for the optimum combination of data from arbitrary magnetic resonance phased array probes," Physics in Medicine and Biology, vol. 47, no. 2, pp. N39-N46, 2002.

[13] U. Dydak, M. Weiger, K. P. Pruessmann, D. Meier, and P. Boesiger, "Sensitivity-encoded spectroscopic imaging," Magnetic Resonance in Medicine, vol. 46, no. 4, pp. 713-722, 2001.

[14] D. Xu, A. P. Chen, C. Cunningham, J. A. Osorio, S. J. Nelson, and D. B. Vigneron, "Spectroscopic imaging of the brain with phased-array coils at 3.0 T," Magnetic Resonance Imaging, vol. 24, no. 1, pp. 69-74, 2006.

[15] J. Keeler, "Elimination of truncation artifacts from NMR spectra. Application to carbon-13 multiplicity determination by two-dimensional spectroscopy," Journal of Magnetic Resonance, vol. 56, no. 3, pp. 463-470, 1984.

[16] Z. Dong, W. Dreher, and D. Leibfritz, "Toward quantitative short-echo-time in vivo proton MR spectroscopy without water suppression," Magnetic Resonance in Medicine, vol. 55, no. 6, pp. 1441-1446, 2006.
[17] H. Barkhuijsen, R. de Beer, and W. M. M. J. Bovee, "Communication: application of linear prediction and singular value decomposition (LPSVD) to determine NMR frequencies and intensities from the FID," Magnetic Resonance in Medicine, vol. 2, no. 1, pp. 86-89, 1985.

[18] G. Goelman, S. Liu, D. Hess, and O. Gonen, "Optimizing the efficiency of high-field multivoxel spectroscopic imaging by multiplexing in space and time," Magnetic Resonance in Medicine, vol. 56, no. 1, pp. 34-40, 2006.

[19] R. Otazo, S. Y. Tsai, F. H. Lin, and S. Posse, "Accelerated shortTE 3D proton echo-planar spectroscopic imaging using 2DSENSE with a 32-channel array coil," Magnetic Resonance in Medicine, vol. 58, no. 6, pp. 1107-1116, 2007.

[20] A. Van Den Boogaart, M. Ala-Korpela, F. A. Howe, L. M. Rodrigues, M. Stubbs, and J. R. Griffiths, "Magnetic resonance spectroscopy data analysis: time or frequency domain?" Magnetic Resonance Materials in Physics, Biology, and Medicine, vol. 2, no. 3, pp. 479-482, 1994.

[21] C. Elster, A. Link, F. Schubert, F. Seifert, M. Walzel, and H. Rinneberg, "Quantitative MRS: comparison of time domain and time domain frequency domain methods using a novel test procedure," Magnetic Resonance Imaging, vol. 18, no. 5, pp. 597-606, 2000.

[22] J. B. Poullet, D. M. Sima, and S. Van Huffel, "MRS signal quantitation: a review of time- and frequency-domain methods," Journal of Magnetic Resonance, vol. 195, no. 2, pp. 134-144, 2008.

[23] R. Bartha, D. J. Drost, and P. C. Williamson, "Factors affecting the quantification of short echo in-vivolH MR spectra: prior knowledge, peak elimination, and filtering," NMR in Biomedicine, vol. 12, no. 4, pp. 205-216, 1999.

[24] S. Mierisova and M. Ala-Korpela, "MR spectroscopy quantitation: a review of frequency domain methods," NMR in Biomedicine, vol. 14, no. 4, pp. 247-259, 2001.

[25] L. Vanhamme, T. Sundin, P. Van Hecke, and S. Van Huffel, "MR spectroscopy quantitation: a review of time-domain methods," NMR in Biomedicine, vol. 14, no. 4, pp. 233-246, 2001. 

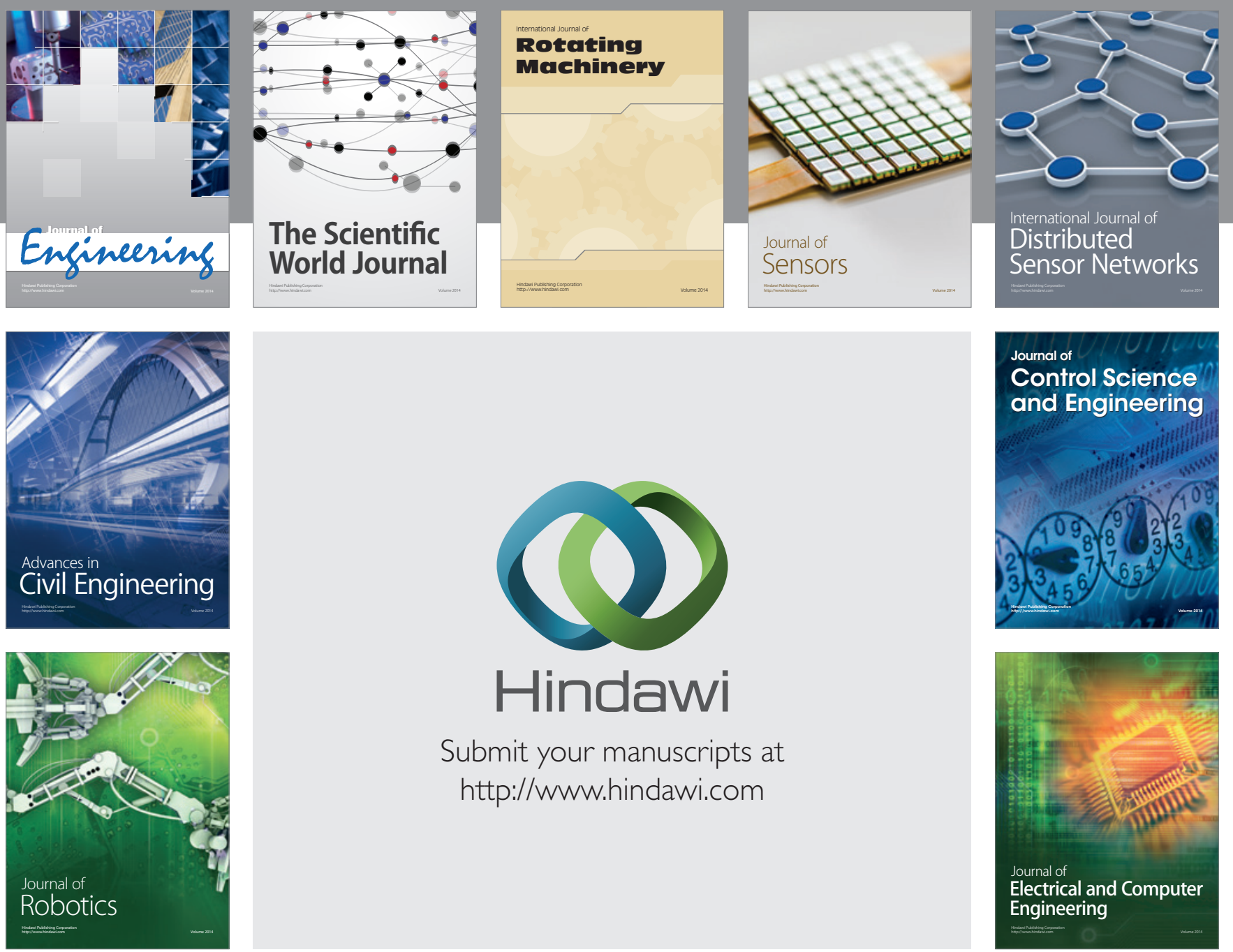

Submit your manuscripts at

http://www.hindawi.com
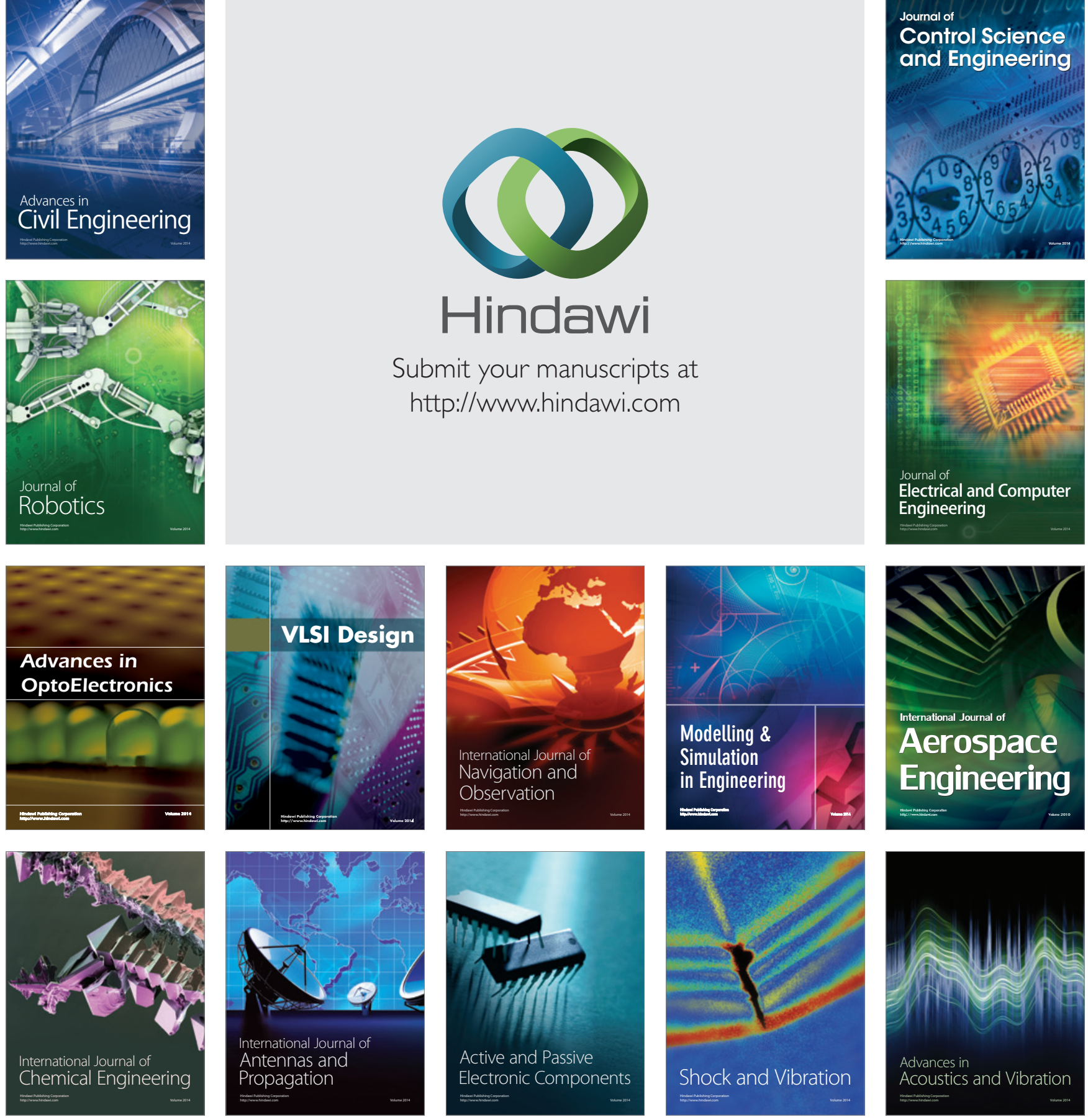